\title{
Screening children with NF1 for optic pathway glioma - No
}

Eye (2010) 24, 1432-1434; doi:10.1038/eye.2010.94;

published online 25 June 2010

This piece aims to outline the areas of uncertainty around the screening for optic pathway glioma (OPG) in children with neurofibromatosis type 1 (NF1). This has been the subject of debate in the United States ${ }^{1}$ and United Kingdom. ${ }^{2}$

Criteria for a valid screening programme can be divided into four areas: the condition, test, treatment, and the screening programme (http://www.gp-training.net/training/ tutorials/management/audit/screen.htm). Each of these will be considered in turn.

(1) The condition should be common enough to justify a screening programme, patients should be identifiable, and there should be a clear natural history.

The 'condition' is OPG in patients with established NF1. OPG are present in 5-25\% of children with NF1. ${ }^{1,3}$ Between 50 and $66 \%$ of OPG do not progress to visual difficulties, and so progressive symptoms are present in $\sim 7.5-15 \%$ of patients with NF1. ${ }^{3}$

The natural history of OPG is not well documented. Most tumours are diagnosed below the age of 7 years, ${ }^{1}$ with a median age of 4.9 years. ${ }^{4}$ Studies looking at the natural history of OPG are complicated by inclusion of children both with and without NF, different modes of detection (MRI, eye screening), and the variability in treatment approaches. However, it can be stated that although there is a range in behaviour from extreme indolence to rapid progression, the majority of tumours are slow growing and, once detected, do not progress radiologically. They rarely metastasise, and exert their symptoms due to compression. ${ }^{3}$

Summary: The condition is common in the defined population, some natural history data exist, and the risks of no treatment include a blind child.

(2) The test should be simple, safe, precise, and validated. The distribution of test values in the target population should be known with a defined cutoff level and agreed policy with regard to further investigations.

Current guidelines as published by the Neurofibromatosis association include a recommendation for visual acuity testing and fundoscopy every year until aged 7 years, ${ }^{5}$ and states that children should have an assessment of colour vision and visual fields 'as soon as the child can co-operate'. The recommendations emphasise that children do not complain of visual impairment.

Data exist for the development of visual acuity in normal individuals. However, in children $<18$ months of age, there is a wide variation in values. Wolsey et $a l^{6}$ found visual acuity testing to have both a sensitivity and specificity of just 50\%. Listernick and co-workers ${ }^{7}$ consider the test not specific for OPG. ${ }^{5}$ There are no visual acuity data for children with NF1 without OPG. Children with NF1 may have reduced vision from the plethora of ophthalmic conditions known to occur in the general population as well as more commonly in NF1. Clinical examination (including visual acuity) yields positive findings in only $20-54 \%$ patients with proven OPG. ${ }^{4,7}$ Fundus examination is possible in all children, although there are no data to suggest that optic atrophy would constitute an early diagnosis. The presence of optic atrophy would suggest that visual acuity would be affected.

There is an erroneous assumption in the guidelines that such testing will exclude OPG. Most OPG do not cause symptoms. Visual acuity testing is only useful if normal or near normal acuity is achieved. There is no guidance on what to do if testing is not possible or not achieved, which may occur given the higher than average prevalence of learning disability in this population.

Neuroimaging of asymptomatic children has not been shown to improve clinical outcomes ${ }^{2}$ nor does early detection reduce the incidence of visual loss due to OPG. ${ }^{3}$ Having a normal 
MRI does not exclude the development of a subsequent tumour. ${ }^{8}$ Where screening using MRI is reported in the literature, there is a high frequency of clinically insignificant 'anomalies' detected on screening images causing anxiety to parents and requiring additional clinical time to explain their significance and neuroimaging for monitoring. ${ }^{9}$

Summary: The test of 'visual acuity testing' fails the screening criteria on many points, but most importantly it is not specific or sensitive. MRI screening is both sensitive and specific, but has not been shown to alter outcomes. MRI under GA carries a small but real risk to the child. It is a precious resource in the NHS and its routine use as a primary screening test needs further evaluation in this context.

(3) The treatment should be effective, and there should be evidence that early treatment has a better outcome than later treatment.

The first course of action once a tumour is identified is radiological and ophthalmological observation. Decision to treat is on the basis of documented progression on imaging and/or clinical signs. Spontaneous regression occurs both radiologically and in terms of visual loss. ${ }^{2,10}$ Tow et $a l^{11}$ followed up a cohort of treated and untreated OPG in NF1. Most had 20/40 or better at 10 years in the better eye, but those who were not treated fared better visually.

Following chemotherapy, tumour progression-free survival is $7 \%$ at 3 years and $50 \%$ at 5 years. ${ }^{12}$ Further details and follow-up are awaited from two multinational studies. However, these data are difficult to interpret in light of the lack of solid data on natural history.

(4) There should be evidence to show that the screening programme is effective. It should be acceptable to patients, families, health-care professionals, and the public. The benefit of the programme should outweigh any physical or psychological harm caused by the test, investigations, or treatment. It should demonstrate value for money.

A review of Manchester patients showed that $17 \%$ of patients are diagnosed with OPG as the presenting feature of NF1 (unpublished data; Sharif S (2004)). Clearly these patients would not benefit from a screening programme for which they were not eligible. A review of all OPG managed by the Manchester paediatric oncology team over 15 years showed that none were picked up during routine ophthalmology screening.

The guidance states that colour vision testing and visual fields be carried out 'as soon as the child can cooperate'. A recent audit of screening practice showed that no children under the age of 7 years could perform these tests adequately. Detection of gross field defects (eg, hemianopia) are possible in young children using confrontational techniques; however, all children with gross field loss due to OPG will also have visual loss ${ }^{13}$ or optic disc pallor. ${ }^{14}$ The same audit showed that over 7 years of screening, no patient was diagnosed with an OPG as a result of a screening visit. This would lead us to question the cost-effectiveness of the screening programme.

\section{Conclusion}

Screening for OPG in children with NF1 fails the criteria on several fronts: most notably the paucity of natural history data, the inadequacy of a test, and the uncertain efficacy of treatment. This is not to say that children with NF1 do not require ophthalmic review: consideration should be given to resources diverted into tests, which may not detect the condition they are being used for, false reassurance given that an ocular disease is absent on the basis of a normal examination, and perhaps most importantly, the justification of yet another day absent from school in a group whose learning may already be impaired.

Finally, those children at highest risk are those who have the lowest sensitivity and specificity from the screening test suggested. Alternative testing such as neuroimaging might lead to earlier diagnosis; however, this needs to be explored as part of a research protocol.

\section{Conflict of interest}

The authors declare no conflict of interest.

\section{References}

1 Listernick R, Ferner R, Liu G, Gurmann D. Optic pathway gliomas in neurofibromatosis-1: controversies and recommendations. Ann Neurol 2007; 61: 189-198.

2 Ferner R. Neurofibromatosis 1 and neurofibromatosis 2: a twenty first century perspective. Lancet Neurol 2007; 6 : 340-351.

3 Listernick R, Louis D, Packer R, Gurmann D. Optic pathway gliomas in children with neurofibromatosis 1: consensus statement from the NF1 optic pathway glioma task force. Ann Neurol 1997; 41(2): 143-149.

4 Lund A, Skovby F. Optic gliomas in children with neurofibromatosis type 1. Eur J Pediatr 1991; 150: 835-838.

5 Ferner R, Huson S, Thomas N, Moss C, Willshaw H, Evans $\mathrm{G}$ et al. Guidelines for the diagnosis and management of individuals with neurofibromatosis. J Med Genet 2007; 44: 81-88.

6 Wolsey DH, Larson SA, Creel D, Hoffman R. Can screening for optic nerve gliomas in patients with neurofibromatosis type I be performed with visual-evoked potential testing? J Am Assoc Pediatr Ophthalmol Strabismus 2006; 10(4): 307-311.

7 King A, Listernick R, Charrow J, Piersall L, Gutmann DH. Optic pathway gliomas in neurofibromatosis type 1: the effect of presenting symptoms on outcome. Am J Med Genet A 2003; 1(122A): 95-99. 
8 Massry GG, Morgan CF, Chung SM. Evidence of optic pathway gliomas after previously negative neuroimaging. Ophthalmology 1997; 104: 930-935.

9 Blazo M, Lewis R, Chintagumpala M, Frazier M, McCluggage C, Plon S. Outcomes of systematic screening for optic pathway tumors in children with neurofibromatosis type 1. Am J Med Genet A 2004; 127A: 224-229.

10 Pepin S, Lessell S. Anterior visual pathway gliomas; the last 30 years. Semin Ophthalmol 2006; 21: 117-124.

11 Tow SL, Chandela S, Miller NR, Avellino AM. Long-term outcome in children with gliomas of the anterior visual pathway. Pediatr Neurol 2003; 2: 262-270.

12 Packer R, Ater J, Allen J. Carboplatin and vincristine chemotherapy for children with newly diagnosed progressive low-grade gliomas. J Neurosurg 1997; 86: 747-754.

13 Listernick R, Charrow J, Gutmann DH. Comments on neuro-fibromatosis 1 and optic pathway tumors. Am J Med Genet A 2001; 102: 105.

14 Kelly JP, Weiss AH. Comparison of pattern visual-evoked potentials to perimetry in the detection of visual loss in children with optic pathway gliomas. J AAPOS 2006; 10 298-306.

$$
\text { RF Pilling }{ }^{1} \text { and RH Taylor }{ }^{2}
$$

${ }^{1}$ Department of Ophthalmology, Manchester Royal Eye Hospital, Manchester, UK

${ }^{2}$ Department of Ophthalmologist, York District Hospital, York, UK

Correspondence: RF Pilling, Department of Ophthalmology, Manchester Royal Eye Hospital,

Oxford Road, Manchester M13 9WL, UK. Tel: + 441612765565 E-mail: rfpilling@btopenworld.com 